\title{
Thoracic endovascular aortic repair for the ascending aorta: experience and pitfalls
}

\author{
Ryan P. Plichta, G. Chad Hughes \\ Department of Surgery, Duke University Medical Center, Durham, NC, USA \\ Contributions: (I) Conception and design: GC Hughes; (II) Administrative support: GC Hughes; (III) Provision of study materials or patients: GC \\ Hughes; (IV) Collection and assembly of data: RP Plichta; (V) Data analysis and interpretation: None; (VI) Manuscript writing: All authors; (VII) \\ Final approval of manuscript: All authors. \\ Correspondence to: G. Chad Hughes. Department of Surgery, Duke University Medical Center, DUMC Box 3051, Durham, NC 27710, USA. \\ Email: Chad.Hughes@Duke.edu.
}

\begin{abstract}
Thoracic endovascular aortic repair (TEVAR) of the ascending aorta is a developing alternative treatment strategy, which currently is specifically aimed at patients who are too high risk for open surgery. TEVAR has been applied to patients with a variety of pathologies of the ascending aorta including type A dissection, intramural hematoma (IMH), penetrating ulcers, aneurysm and pseudoaneurysm. Here we discuss the current evidence regarding the use of TEVAR for the ascending aorta as well as the latest techniques and pitfalls of the procedure. The challenges of this modality are considerable, and the techniques that have been applied draw from the many facets of endovascular experience. There is limited literature regarding the use of stent grafts in the ascending aorta, and the pool of patients currently considered appropriate candidates for the procedure is small. This is an evolving intervention that warrants further study and the development of devices specifically engineered to meet the anatomical and physiologic challenges of the ascending aorta.
\end{abstract}

Keywords: Thoracic endovascular aortic repair (TEVAR); ascending aorta; endovascular; cardiac surgery

Received: 23 November 2017; Accepted: 09 February 2018; Published: 09 May 2018.

doi: 10.21037/jovs.2018.03.01

View this article at: http://dx.doi.org/10.21037/jovs.2018.03.01

\section{Introduction}

Surgical options for pathology of the ascending aorta have traditionally been limited to open approaches, typically via median sternotomy. Patients with prohibitive surgical risk who are not candidates for open procedures may be considered for Thoracic endovascular aortic repair (TEVAR) provided they have appropriate anatomy. There is limited literature regarding the application of stent grafts to ascending aortic surgical pathology, and the use of ascending TEVAR has remained confined to the purview of high volume centers with specialized aortic surgical teams. The reported case series in the literature to date support the feasibility of applying TEVAR to ascending aortic repairs under specific circumstances (1-11).

Open surgical repair of the ascending aorta is performed with excellent outcomes, even in high risk patients in emergent situations. While the number of patients turned down for open surgery has decreased over the past decade, approximately $10 \%$ of patients with an emergent surgical indication such as acute type A dissection are deemed too high risk for open surgery, even at high volume centers (12). In these situations, TEVAR of the ascending aorta can be considered, provided the patient's anatomy lends itself to an endovascular repair. However, there are currently no endovascular devices FDA-approved for use in the ascending aorta. What follows is a review of the reported experience with TEVAR in the ascending aorta, a case report of TEVAR for an ascending aortic pseudoaneurysm, as well as pearls and pitfalls regarding this challenging and developing application of thoracic stent grafting.

\section{Background}

TEVAR to treat the ascending aorta [so-called "zone 0" 

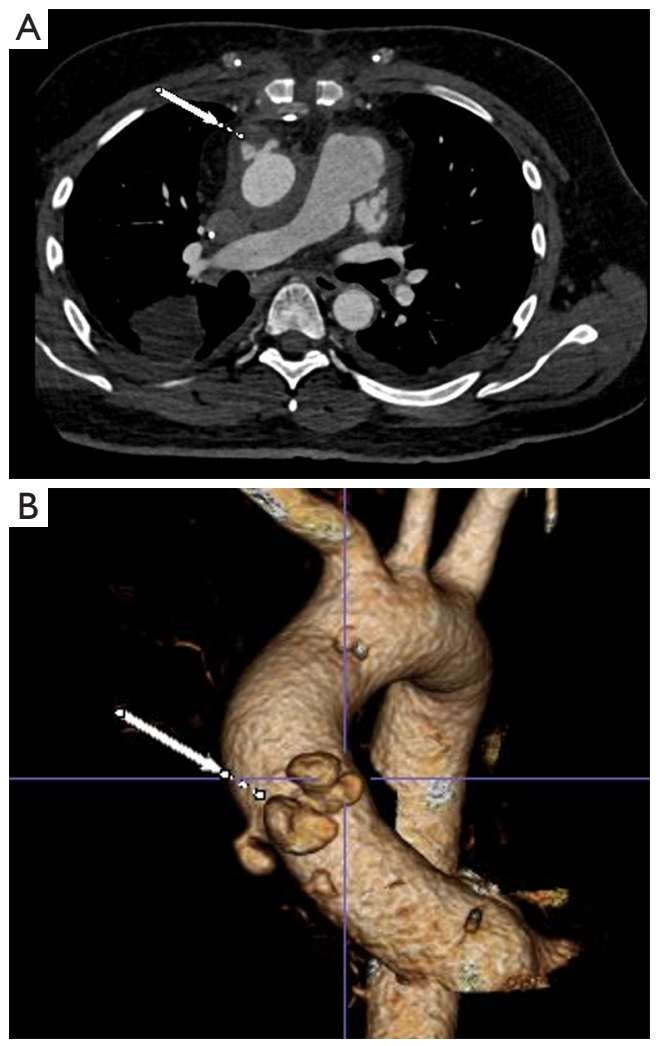

Figure 1 Pre-operative axial (A) and 3D reconstruction (B) CTA images demonstrating large mycotic ascending aortic anastomotic pseudoaneurysm (white arrow) in a 35-year-old male who had undergone previous orthotopic heart transplant.

using the Ishimaru anatomical landing zone map (13)] has been shown to be feasible in patients who are poor conventional open operative candidates. This group of patients present with a variety of ascending pathologies that include type A acute aortic dissection (TAAD), intramural hematoma (IMH), penetrating aortic ulcers (PAU), and aneurysms or ascending aortic pseudoaneurysms (AAP). Reported case series of TEVAR in zone 0 of the aorta are dominated by sick patients with indications for emergency surgery, who are otherwise not candidates for open procedures. Despite this, outcomes have shown TEVAR in the acute setting to be a viable option. Additionally, given favorable anatomy, this approach has been applied to a wide variety of ascending pathology.

With regard to outcomes for TAAD and ascending and arch surgery in general, reports from the International Registry of Acute Aortic Dissection (IRAD) have shown that while outcomes have progressively improved over the last two decades, the operative mortality for TAAD still ranges from $17 \%$ to $26 \%$ (10). Also, approximately $10 \%$ of patients are turned down for surgery when presenting with a TAAD. Williams et al examined the Society of Thoracic Surgeons (STS) database and found that the operative mortality for ascending and arch surgery in North America over the previous decade was $3.4 \%$ for elective cases and $15.4 \%$ for non-elective cases (14). Clearly, open aortic surgery is a safe and viable option for patients who are operative candidates. However, for the group of patients who will not tolerate open surgery and with favorable anatomy, an endovascular approach in the ascending aortic position is becoming a viable option.

\section{Case report}

We present a case of mycotic ascending aortic pseudoaneurysm in a patient who was very high risk for an open surgical approach. This was a 35 -year-old male who had previously undergone orthotopic heart transplant for non-ischemic cardiomyopathy earlier in the year. This index case was complicated by a candidal deep sternal wound infection that resulted in multiple re-operations involving sternal debridements and ultimately an omental flap for tissue coverage. The patient recovered from these procedures and his candidal infection was treated with dual antifungal therapy for 2 months; his most recent blood cultures were negative. Unfortunately, the patient subsequently presented with signs of a stroke as well as a sentinel bleed from a mediastinal drain that was placed at the time of flap coverage. He was noted on serial imaging to have developed a pseudoaneurysm at the ascending aortic anastomotic site that was rapidly expanding and currently measured $4.8 \mathrm{~cm}$ (Figure 1). The patient was extremely debilitated following his prolonged illness and recent stroke and felt to be a poor surgical candidate for a multiple redosternal exploration and open proximal aortic replacement utilizing cardiopulmonary bypass.

The aortic team was subsequently consulted, and his anatomy was felt to be optimal for placement of an aortic endograft in the ascending position. On thin-cut computed tomography angiography (CTA), the location of the AAP was measured to be $3 \mathrm{~cm}$ above the sino-tubular junction (STJ) and would allow for at least a $1 \mathrm{~cm}$ distal landing zone just proximal to the origin of the innominate artery. Given his history of heart transplant, his ascending aorta was considerably long with the AAP located at the donor to native aorta anastomotic site. 


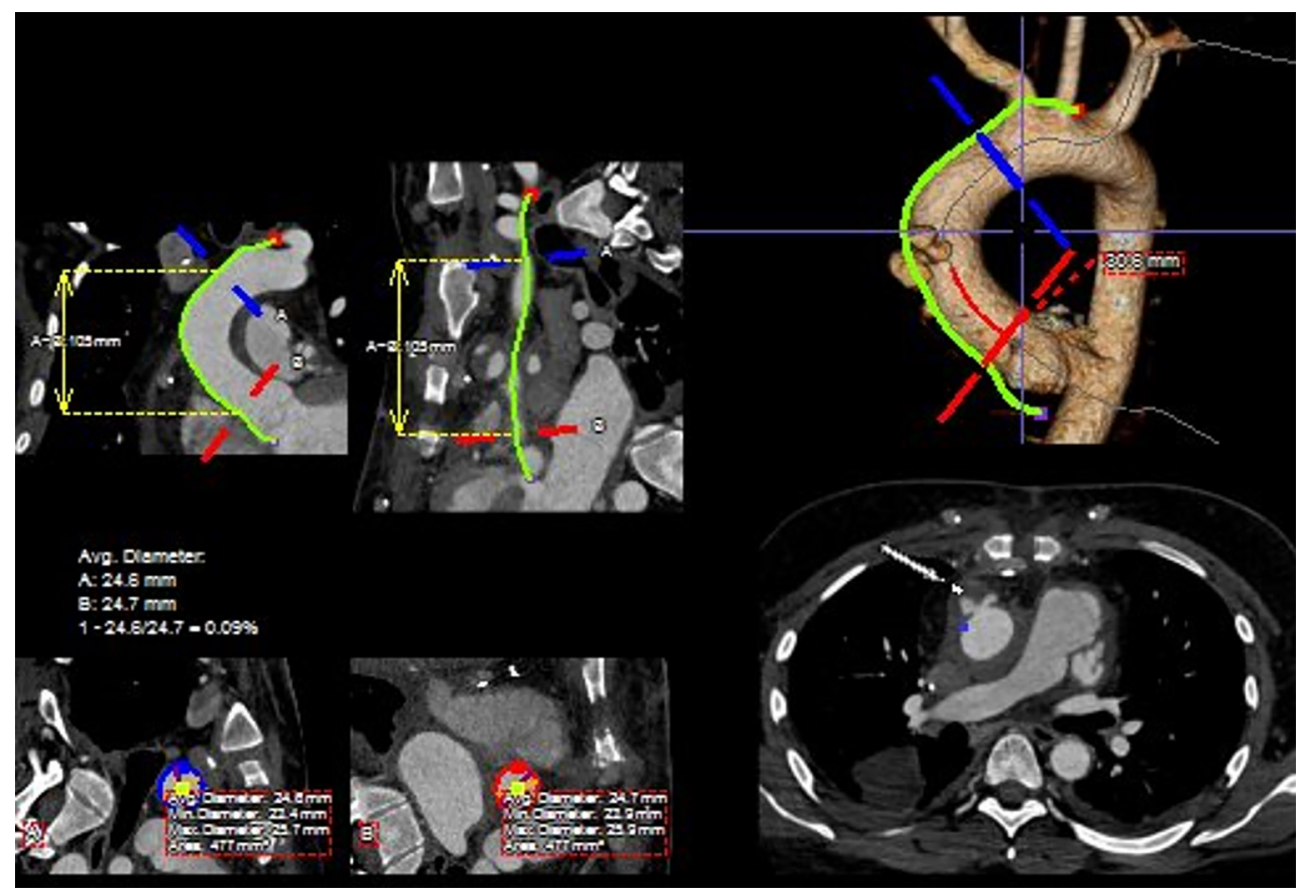

Figure 2 Curved planar reformats with centerline reconstruction of the ascending aorta from the 3D CTA with corresponding measurements of landing zone diameters and centerline and greater curvature lengths. CTA, computed tomography angiography.

\section{Pre-operative preparation}

Detailed image analysis was performed with the use of 3-dimensional imaging software (Terarecon, San Mateo, CA, USA) review of the thin slice CTA scan. The scan was reviewed and the AAP was identified at the aortic anastomosis site, approximately $3 \mathrm{~cm}$ distal to the STJ and $5 \mathrm{~cm}$ proximal to the takeoff of the innominate artery. The total distance measured along the greater curve of the aorta was $12 \mathrm{~cm}$, facilitating the use of a commercially available $10 \mathrm{~cm}$ length thoracic endograft (Figure 2).

Given the size of the AAP, rapid growth, and sentinel bleed, the decision was made to proceed with urgent TEVAR of the ascending aorta to exclude the anastomotic pseudoaneurysm.

\section{Procedure}

The procedure was performed in a hybrid operating room with continuous trans-esophageal echocardiographic (TEE) monitoring with a dedicated cardiac anesthesiology team. This team placed invasive monitoring lines including bilateral radial arterial lines and a central venous line in the left internal jugular vein. Preoperative antibiotics were administered and a surgical time out was performed. The patient was prepped and draped from the chin to midthighs.

A $3 \mathrm{~cm}$ incision was made along the anterior border of the right sternocleidomastoid muscle, and the dissection carried down through the platysma. The right common carotid artery was dissected free for a length of several centimeters and accessed with a micro-puncture needle; a micro-wire was then placed via this needle down into the aortic arch to facilitate identification of the origin of the innominate artery under fluoroscopy.

A $5 \mathrm{~cm}$ incision was made at the level of the inguinal ligament on the left and a \#7-French sheath placed into the left common femoral artery. The right common femoral artery was accessed percutaneously using a micro-puncture technique under ultrasound guidance, and a \#5-French sheath placed. The left common femoral vein was accessed, and a \#6-French pacing sheath placed. The patient was systemically heparinized with 100 units per $\mathrm{kg}$ of IV heparin to a goal ACT of $>200$. A transvenous pacing lead was then advanced from the left femoral venous sheath up to the right ventricle and capture was confirmed.

A marker pigtail catheter was passed up from the right leg and positioned in the non-coronary sinus. The aortic valve was then crossed using a straight 6 -Fr pigtail catheter 


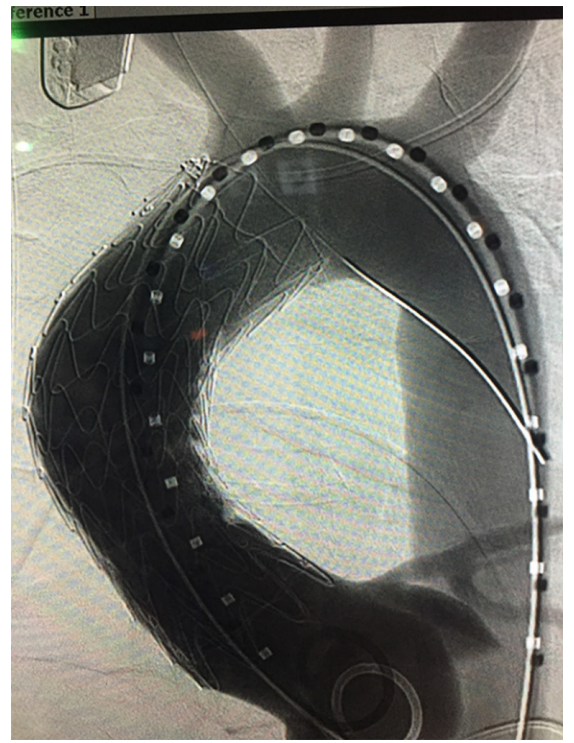

Figure 3 Intraoperative completion angiogram following successful deployment of the CTAG device in the ascending aortic position.

passed up from the left leg. A Boston Scientific Safari-2 wire (Boston Scientific Corporation, Marlborough, MA, USA) with a pre-fashioned curve was positioned in the left ventricular apex.

The \#7-French sheath was then exchanged for a \#22-French Gore DrySeal introducer sheath (W. L. Gore \& Associates, Flagstaff, Ariz, USA). The C-arm was then placed in slight left anterior oblique (LAO)/caudal angulation to lay out the aortic root in a co-planar view. A marker thoracic arteriogram was shot to road-map the ascending aorta and proximal arch. A second angiogram was shot during rapid ventricular pacing to a rate of 180 , which demonstrated minimal movement of the ascending aorta.

A conformable TAG (C-TAG) (W. L. Gore \& Associates, Flagstaff, Ariz, USA) $31 \mathrm{~mm} \times 10 \mathrm{~cm}$ device was passed up from the left groin and positioned with the proximal end $1 \mathrm{~cm}$ above the STJ and the distal end just proximal to the innominate artery, and with long segment seal on either side of the easily visualized pseudoaneurysm. The device was then deployed and seated nicely in the intended position.

A completion thoracic arteriogram demonstrated no endoleak and complete exclusion of the pseudoaneurysm, as well as good flow in both coronary arteries and all of the arch vessels (Figure 3). Additionally, there was no aortic insufficiency (AI) by TEE after device deployment.

The patient tolerated the procedure well and serial
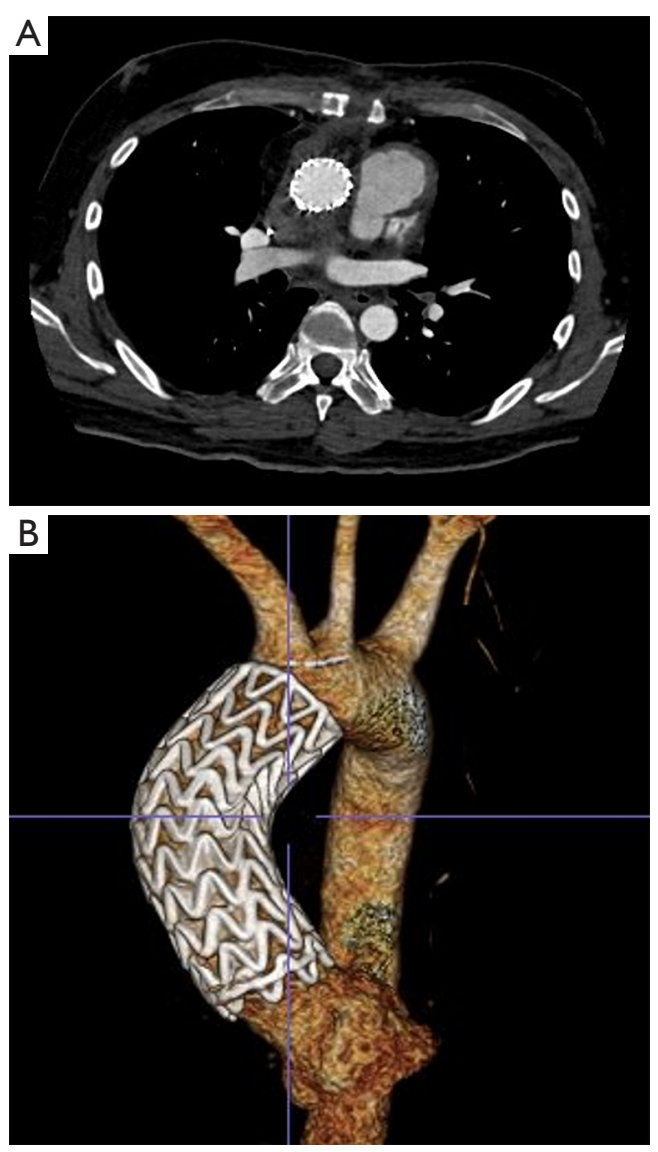

Figure 4 Follow up axial (A) and 3D reconstruction (B) CTA imaging status-post TEVAR of the ascending aorta demonstrating complete resolution of the mycotic pseudoaneurysm with no endoleak. CTA, computed tomography angiography.

imaging of the ascending aorta revealed complete resolution of the AAP (Figure 4). Given the mycotic nature of the aneurysm and the patient's immunocompromised state, the patient will remain on lifelong antibiotic therapy.

\section{Review of experience}

Although this is a very limited patient population, the existing case reports have demonstrated an impressive record of success with TEVAR in the ascending aorta. Patient selection is of paramount importance as the ascending aorta poses significant anatomic and hemodynamic challenges. The position is complicated by the location of the coronary arteries, the shear stresses and hemodynamics of the left ventricular outflow tract forces, the curvature of the ascending aorta, and the position and 
proximity of the arch vessels. All of these factors must be considered when selecting patients.

A recent comprehensive review of the literature by Muetterties et al. revealed excellent outcomes in this high risk subset of patients as well as, unsurprisingly, a lack of standardized technique. In their review that looked at the published experience from January 1, 1995 to January 31, 2017, they found reports on 118 patients who underwent TEVAR of the ascending aorta. The most commonly used device was a thoracic stent graft, $71.2 \%$; extender cuffs were used in $11 \%$. TAAD was the most common indication for surgery at $50 \%$, followed by AAP (29.7\%), with the remainder treating aneurysms, PAU and acute aortic rupture. Transfemoral access was most common, used in $62.7 \%$ of cases, followed by transapical (14.4\%), trans-carotid (12.7\%), and trans-axillary (6.8\%). The rate of type 1 endoleak was $18.6 \%$, with $9.3 \%$ requiring reintervention. The all-cause mortality was $15.2 \%$, the aortarelated mortality was $5 \%$, and the average follow-up was 17.2 months (11). Given the high risk patient population and the emergent indication of TAAD in half of the reported cases, these results are impressive.

In the series from Roselli et al., the authors examined their experience with TEVAR of the ascending aorta in 22 patients over 8 years. These were all high risk patients who were deemed inoperable and agreed to undergo off-label treatment with TEVAR. Despite the high risk population and relatively experimental nature of the approach, the inhospital mortality was only $13.6 \%$. The 30 -day, 1 and 5 -year survivals were $86 \%, 80 \%$, and $75 \%$, respectively. Multiple endovascular devices were employed over the course of the study and via several different access sites (6).

Similarly, Preventza et al. demonstrated success with this approach in seven patients over 7 years with a similar 30 -day mortality of $14.3 \%$. They achieved technical success in all but one patient and had no incidence of stroke and a 1 -year survival of $66.6 \%$. The majority of their patients were treated for an AAP, while one was treated for an iatrogenic coarctation, and all were considered high risk for open surgery and otherwise not operative candidates (15).

Vallabhajosyula et al. had six patients over 7 years who underwent TEVAR of the ascending aorta: two patients had TAAD while four of had AAP. All were considered high risk for open surgery. They reported an $83 \%$ success rate, with $0 \%$ in-hospital and 30-day mortality, one minor stroke and one endoleak. They too used multiple different devices and a variety of access strategies (7).

There are several case reports that focused specifically on ascending aortic pseudoaneurysm and penetrating ulcers $(1,2,9)$. These include both infected and noninfected pseudoaneurysms. Applying a stent graft repair to a mycotic aneurysm, as in our case report, is a controversial approach that is frequently born out of a lack of options in considerably moribund patients. There is an obvious risk of seeding the graft with the infection, and long term antibiotic therapy is required. Piffaretti et al. looked specifically at AAP and PAU. This series included one mycotic AAP that was successfully treated with TEVAR, and the patient remained on lifelong antibiotic therapy. Overall they found an $87 \%$ success rate with only 1 endoleak that was managed expectantly. The in-hospital mortality was $0 \%$, they had no conversions to open surgery, no valve or coronary impairments, no strokes and no reinterventions (9).

\section{Patient selection}

With the current technology available, TEVAR of the ascending aorta is not an option for every high or extreme risk patient. Multiple anatomic considerations must be evaluated and all patients must undergo CTA of the entire aorta for successful operative planning. As with any endovascular stent grafting procedure, there must be adequate access for device delivery as well as suitable proximal and distal landing zones. Strict criteria for stent grafting the ascending aorta have been suggested and recommend at least $1 \mathrm{~cm}$ of proximal and distal landing zone and STJ diameters under $38 \mathrm{~mm}$ (16). This is particularly important with degenerative aneurysms and may be less of a concern with focal lesions. AAP, PAU, $\mathrm{IMH}$, as well as some TAAD can be successfully covered and treated with less than the $2 \mathrm{~cm}$ of landing zone normally required for TEVAR. The repair, however, can only address pathology above the STJ to avoid the coronary ostia and aortic valve, and the distal extent must be proximal to the take-off of the arch vessels. Consideration can be given to partial coverage of the innominate artery, as well as extra-anatomic arch debranching, to achieve an adequate distal seal zone when the lesion is very close to the arch vessels. Aneurysmal dilation of the ascending aorta can also impair the ability to get an adequate seal and may preclude the use of ascending TEVAR. Patent bypass grafts on the ascending aorta from previous coronary artery bypass surgery are also a contraindication for TEVAR of the ascending aorta, as coverage would result in occlusion and ischemia. Severe AI, as is frequently seen in TAAD, is not a contraindication for the use of TEVAR. Roselli et al. 
found that the AI frequently improves or resolves when the dissection is treated and the STJ and commissures return to their anatomic positions (6).

\section{Device selection and access}

There are several endovascular devices that can be used in the ascending position, the majority of which are not specifically designed to meet the challenges of this anatomical region. Thoracic or infrarenal aortic extender cuffs can be a useful tool when the length of the ascending aorta is short. Frequently, more than one cuff is required to achieve an adequate seal. The delivery systems on the infrarenal devices are typically too short to be deployed via transfemoral access and alternative access is preferred. Trans-carotid or trans-axillary device deployment is more appropriate with these cuffs and can also help with accurate placement, given the shorter distance from the access site to the target region, which is of critical importance with such limited real estate. Thoracic stent grafts are also useful when the length of the ascending aorta can accommodate a $10 \mathrm{~cm}$ length device, as in the case report herein. These thoracic grafts have improved conformability that lends itself nicely to the curvature of the ascending aorta. Also, these devices have longer delivery systems that allow for deployment via transfemoral access. Transapical access is another consideration, however we rarely find this approach necessary.

Currently, there are no FDA-approved devices for use in the ascending aorta. There are some devices being tested and the results have, thus far, been promising. Tsilimparis et al. recently reported a series of patients treated with a device designed specifically for use in the ascending aorta, the Zenith Ascend TAA Endovascular Graft (William Cook Europe, Bjaeversklov, Denmark) (10). They treated 10 patients who were high risk for open surgery with this device, and all endografts were deployed successfully without intraoperative complications. The 30-day mortality rate was $10 \%$. They had 1 transient ischemic attack, 1 patient with a stroke and paraplegia, 2 late reinterventions for persistent endoleak, and 3 late deaths. Nearly half of these procedures were done for emergent indications. The Zenith device has been designed with the challenges of the ascending aorta in mind. It is a single component device constructed with a woven polyester fabric sewn to a self-expanding nitinol frame with bare stent material both proximally and distally. It is produced in diameters ranging from 28 to $46 \mathrm{~mm}$ and has a covered length of
$65 \mathrm{~mm}$. It also has a ring of radio-opaque markers on both the proximal and distal ends indicating the location of the extent of graft material for more precise placement. The device is deployed via a 16,18 , or $20 \mathrm{~F}$ delivery system that is $100 \mathrm{~cm}$ in length and also allows for fine positional adjustments after retraction of the sheath.

\section{Device sizing and deployment}

Sizing of the endograft is of critical importance. Measurements are made using CTA imaging in the major axis in the cross section and perpendicular to the centerline of flow (Figure 2). Given the hemodynamic forces affecting the ascending aorta, the diameter can vary greatly during the cardiac cycle, and it is important to oversize the device according to the instructions for use (IFU) of the endograft. It has been recommended to oversize the graft by $20 \%$ in chronic conditions, while it may be advisable to oversize by only $10 \%$ with an acute pathology and to account for the friability of the tissue $(6,8)$. The length of the ascending aorta is also of concern, and we typically use the length of the greater curvature as our treatment length measurement (Figure 2). Partial coverage of the innominate artery is acceptable, provided this is not flow limiting. Consideration can also be given to extra-thoracic bypass of the arch vessels to accommodate a device when the distal landing zone requires some coverage of the head vessels. As side branch devices become available, the options for intervening on the ascending aorta and arch will increase considerably.

The addition of extra-thoracic bypass grafting introduces the added risk of a concomitant surgical procedure which must be weighed against the potential benefit to prohibitive risk patients who would otherwise have no surgical or endovascular options, although in our experience cervical arch debranching procedures via neck incisions are generally well tolerated (17). Figure 5 demonstrates the use of an investigational single side-branched endograft deployed following right common carotid to left common carotid to left subclavian artery cervical arch debranching bypass to treat a saccular chronic post-traumatic transverse arch pseudoaneurysm in a high risk patient.

Ascending aortic device deployment requires significant accuracy given the limited length of the landing zones in this section of the aorta. The proximity of the coronary ostia is of critical concern and coverage needs to be avoided at all costs. Similar to transcatheter aortic valve replacement in the setting of small aortic root diameter or low coronary height, obtaining pre-deployment wire access of the coronary 

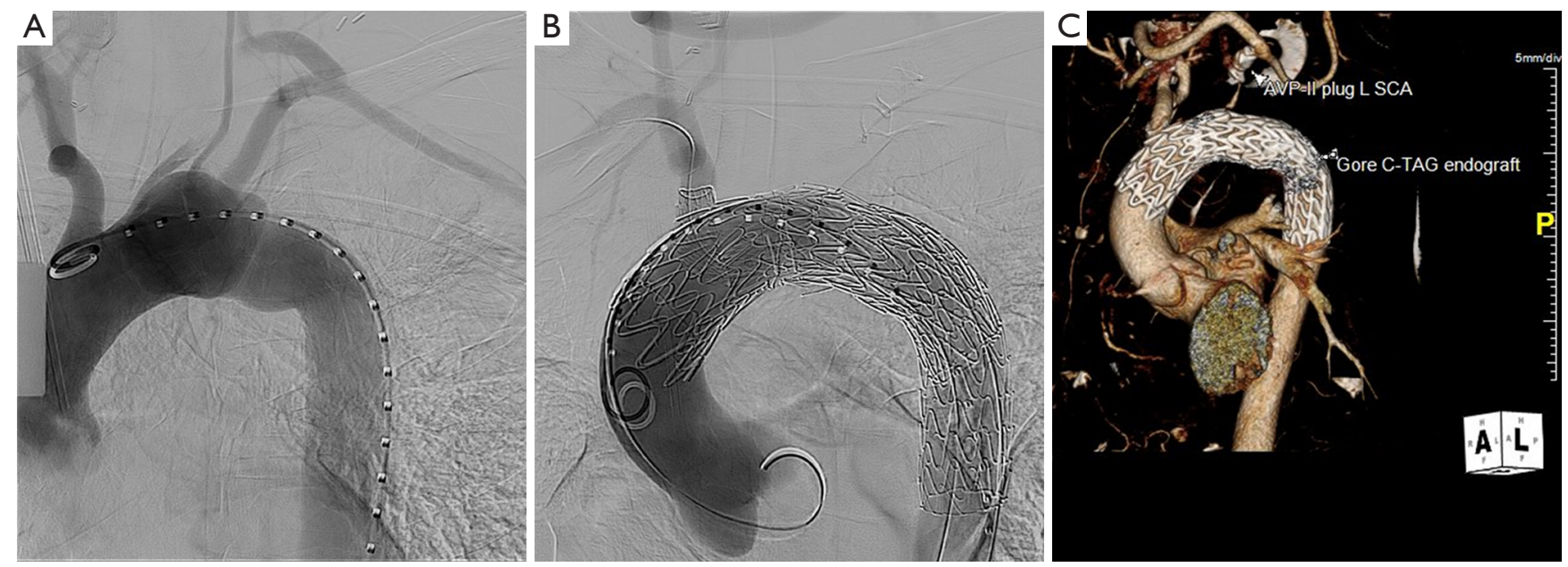

Figure 5 Pre (A) and post (B) deployment angiograms of a single side-branched endograft used to treat a saccular chronic post-traumatic transverse arch pseudoaneurysm in a high-risk patient following extra-thoracic cervical arch debranching. Follow up 3D CTA reconstruction (C) demonstrating widely patent right carotid-left subclavian bypass with endovascular occlusion plug in proximal left subclavian artery and well positioned aortic and side branch endografts without endoleak. CTA, computed tomography angiography.

ostia is recommended prior to ascending TEVAR when the landing zone abuts the STJ, although this precautionary measure does not ensure successful placement of the stent graft. If accidental coronary coverage does occur, emergent conversion to an open procedure may be necessary. Alternatively, there are reports of endovascular rescue by inflating an endo-balloon within the device and allowing the heart to eject against it. This may pull the endograft back above the coronary ostia and should be attempted as a firstline maneuver in this difficult scenario (6).

Rapid ventricular pacing can aid in more accurate delivery of the device by limiting the movement of the landing zone and decreasing the forces applied to the stent graft by reducing the cardiac output. However, not all ventricles can tolerate rapid pacing and, rarely, it may be advisable to place the patient on cardiopulmonary bypass preemptively. When the landing zone is in the mid ascending aorta and the treatment length is short, rapid pacing is not always required.

\section{Conclusions}

TEVAR of the ascending aorta is a developing modality without commercially available devices designed specifically for this use, and without standard operating procedures or practice guidelines. Despite these limitations, several groups, including our own, have demonstrated its feasibility in properly selected patients. The reported series have demonstrated impressive outcomes even in emergent, rescue scenarios with patients thought to be otherwise inoperable (1-11).

Clearly, the gold standard for treatment of ascending aortic pathology is open repair, with excellent outcomes despite often high risk patients undergoing emergency surgery. However, there are patients who have prohibitive surgical risks and are treated with medical management with subsequent high mortality. In a previously reported IRAD series, up to $28 \%$ of acute type A dissection patients were found to be unfit for surgery (18). Although that rate has decreased to $10-15 \%$ over the last decade, this still represents a significant number of patients. This cohort, along with other inoperable patients who have surgical ascending aortic pathology such as pseudoaneurysm and focal chronic dissection, represent a group who can be considered for ascending aorta endograft repair when there is appropriate anatomy.

Several factors have emerged as keys to success in this procedure. The most important factor is patient selection. The patient must have anatomy that lends itself to an endovascular approach. There must be at least $1 \mathrm{~cm}$ of proximal and distal landing zone that does not compromise the coronary ostia. The ascending aorta must accommodate an endograft within the treatment sizing range, and there must be a distal landing zone that does not compromise the arch vessels. Additionally, there cannot be previous coronary bypass grafts that would be occluded with coverage of the 
ascending aorta.

Devices designed with the challenges inherent to the ascending aorta and its associated pathologies in mind are critical to the development and utilization of this approach. With the advent of side branch devices, as well as emerging stent grafts with increased conformability, longer delivery systems, and a variety of treatment lengths in the range of 5-8 cm, TEVAR of the ascending aorta may become a viable option for those patients who cannot tolerate open surgery.

\section{Acknowledgements}

None.

\section{Footnote}

Conflicts of Interest: The authors have no conflicts of interest to declare.

Informed Consent: Written informed consent was obtained from the patient for publication of this manuscript and any accompanying images.

\section{References}

1. Gelpi G, Cagnoni G, Vanelli P, et al. Endovascular repair of ascending aortic pseudoaneurysm in a high-risk patient. Interact Cardiovasc Thorac Surg 2012;14:494-6.

2. Gray BH, Langan EM 3rd, Manos G, et al. Technical strategy for the endovascular management of ascending aortic pseudoaneurysm. Ann Vasc Surg 2012;26:734-8.

3. Metcalfe MJ, Karthikesalingam A, Black SA, et al. The first endovascular repair of an acute type A dissection using an endograft designed for the ascending aorta. J Vasc Surg 2012;55:220-2.

4. Lu Q, Feng J, Zhou J, et al. Endovascular repair of ascending aortic dissection: a novel treatment option for patients judged unfit for direct surgical repair. J Am Coll Cardiol 2013;61:1917-24.

5. Bernardes RC, Navarro TP, Reis FR, et al. Early experience with off-the-shelf endografts using a zone 0 proximal landing site to treat the ascending aorta and arch. J Thorac Cardiovasc Surg 2014;148:105-12.

6. Roselli EE, Idrees J, Greenberg RK, et al. Endovascular stent grafting for ascending aorta repair in high-risk patients. J Thorac Cardiovasc Surg 2015;149:144-51.

7. Vallabhajosyula P, Gottret JP, Bavaria JE, et al. Endovascular repair of the ascending aorta in patients at high risk for open repair. J Thorac Cardiovasc Surg 2015;149:S144-50.

8. Li Z, Lu Q, Feng R, et al. Outcomes of Endovascular Repair of Ascending Aortic Dissection in Patients Unsuitable for Direct Surgical Repair. J Am Coll Cardiol 2016;68:1944-54.

9. Piffaretti G, Galli M, Lomazzi C, et al. Endograft repair for pseudoaneurysms and penetrating ulcers of the ascending aorta. J Thorac Cardiovasc Surg 2016;151:1606-14.

10. Tsilimparis N, Debus ES, Oderich GS, et al. International experience with endovascular therapy of the ascending aorta with a dedicated endograft. J Vasc Surg 2016;63:1476-82.

11. Muetterties CE, Menon R, Wheatley GH 3rd. A systematic review of primary endovascular repair of the ascending aorta. J Vasc Surg 2018;67:332-42.

12. Berretta P, Patel HJ, Gleason TG, et al. IRAD experience on surgical type A acute dissection patients: results and predictors of mortality. Ann Cardiothorac Surg 2016;5:346-51.

13. Fillinger MF, Greenberg RK, McKinsey JF, et al. Reporting standards for thoracic endovascular aortic repair (TEVAR). J Vasc Surg 2010;52:1022-33,1033.e15.

14. Williams JB, Peterson ED, Zhao Y, et al. Contemporary results for proximal aortic replacement in North America. J Am Coll Cardiol 2012;60:1156-62.

15. Preventza O, Henry MJ, Cheong BY, et al. Endovascular repair of the ascending aorta: when and how to implement the current technology. Ann Thorac Surg 2014;97:1555-60.

16. Moon MC, Greenberg RK, Morales JP, et al. Computed tomography-based anatomic characterization of proximal aortic dissection with consideration for endovascular candidacy. J Vasc Surg 2011;53:942-9.

17. Andersen ND, Williams JB, Hanna JM, et al. Results with an algorithmic approach to hybrid repair of the aortic arch. J Vasc Surg 2013;57:655-67; discussion 66-7.

18. Hagan PG, Nienaber CA, Isselbacher EM, et al. The International Registry of Acute Aortic Dissection (IRAD): new insights into an old disease. JAMA 2000;283:897-903.

doi: $10.21037 /$ jovs.2018.03.01

Cite this article as: Plichta RP, Hughes GC. Thoracic endovascular aortic repair for the ascending aorta: experience and pitfalls. J Vis Surg 2018;4:92. 